\title{
OA03-06 LB. Unique stimulatory properties of myeloid dendritic cells in individuals with "elite" HIV-I control
}

\author{
J Huang*2, L Poole ${ }^{2}$, P Burke ${ }^{2}$, T Cung ${ }^{2}$, A Trocha ${ }^{2}$, F Pereyra ${ }^{2}$, L Borges ${ }^{1}$, \\ $\mathrm{M}$ Lichterfeld ${ }^{2}$ and $\mathrm{X} \mathrm{Yu}^{2}$
}

Address: ${ }^{1}$ Amgen Inc, Seattle, WA, USA and ${ }^{2}$ Massachusetts General Hospital, Boston, MA, USA

* Corresponding author

from AIDS Vaccine 2009

Paris, France. 19-22 October 2009

Published: 22 October 2009

Retrovirology 2009, 6(Suppl 3):O24 doi:10.I I86/I742-4690-6-S3-O24

This abstract is available from: http://www.retrovirology.com/content/6/S3/O24

(c) 2009 BioMed Central Ltd.

\section{Background}

"Elite" controllers are HIV-1 infected patients who spontaneously maintain undetectable levels of HIV-1 viremia. The Identification of unique immunological characteristics in this specific patient population is a premier opportunity for determining components of immune protection against HIV-1.

\section{Methods}

Functional properties of freshly isolated myeloid dendritic cells were analyzed in mixed lymphocyte reactions. Surface expression of costimulatory molecules and myelomonocytic receptors was analyzed by multiparameter flow cytometry. Targeted si-RNA mediated gene knockout was used to determine the impact of specific functional effects mediated by regulatory myelomonocytic receptors. Assays were performed in elite controllers $(n=11)$ and HIV-1 progressors $(n=11)$ or healthy HIV-1 uninfected persons $(n=12)$ as reference cohorts.

\section{Results}

In comparison to both HIV-1 progressors or healthy persons, dendritic cells from "elite" controllers had significantly higher ability to expand allogeneic CD4+ and CD8+ T cells. Costimulatory molecules (CD80, CD86, CD40, CD83) were expressed at higher levels in HIV-1 progressors, while no significant difference was observed between controllers and HIV-1 negative persons. To determine underlying reasons for the increased functional activity of mDCs in controllers, we assessed the expression of regulatory dendritic cells receptors that can critically modulate dendritic cell function. The receptor ILT2 and ILT5 were significantly higher expressed on $\mathrm{mDC}$ in controllers, as opposed to either progressors of HIV-1 negative persons. Si-RNA mediated knock out of ILT2 or ILT5 led to significant reduction of the functional activity of dendritic cells, indicating that it has a critical stimulatory effect on these cells.

\section{Conclusion}

HIV-1 "elite" controllers have myeloid dendritic cells with a uniquely increased allostimulatory activity. This extraordinary functional profile is mediated by signaling through ILT2 and ILT5, which are uniquely upregulated on these cells. Manipulation of dendritic cell function might thus represent a promising strategy for immunological approaches to treatment and prevention of HIV-1 infection. 